\title{
La Presión Arterial Normal Alta está asociada a un mayor riesgo de enfermedad cardiovascular
}

Impact of high-normal blood pressure on the risk of cardiovascular disease.Vasan RS, Larson M, Leip E y cols. New Engl J Med. 2001; 345: 1291-1297.

\section{Objetivo}

Investigar el riesgo de eventos cardiovasculares en pacientes con Presión Arterial Normal Alta (Presión Arterial Sistólica de 130 a 139 mm Hg, Presión Arterial Diastólica de 80 a 89 mm Hg, o ambas)

Diseño

Estudio de cohortes* (revisión banco de datos del Framingham Heart Study)

Lugar

Comunidad de Framingham, EE.UU

\section{Pacientes}

Se incluyeron en el estudio 6.859 pacientes de ambos sexos ( $43 \%$ hombres), mayores de 34 años de edad, inicialmente sin hipertensión arterial y libres de enfermedad cardiovascular.

\section{Evaluación de Factores Pronósticos}

Se categorizó a los pacientes de acuerdo a su Presión Arterial (PA) en: PA Optima (PA < $120 / 80: 42 \%$ de los pacientes); PA Normal (PA 120 a 129 / 80 a 84 : 32\%); y PA Normal Alta (PA 130 a 139 / 85 a $89: 26 \%$ de la población). Se relevaron además otras variables como colesterolemia, tabaquismo, índice de masa corporal (BMI) y diabetes mellitus.

\section{Medición de Resultados Principales}

Los pacientes eran monitoreados para eventos cardiovasculares y muerte. El resultado principal era el tiempo transcurrido hasta la ocurrencia del primer evento mayor: muerte por enfermedad cardiovascular, infarto agudo de miocardio (IAM), accidente cerebrovascular (ACV) o insuficiencia cardíaca (ICC).

\section{Resultados Principales}

Después de un seguimiento promedio de 11 años, 397 pacientes (138 mujeres y 259 hombres) tuvieron un primer evento cardiovascular, incluyendo 42 muertes, $190 \mathrm{IAM}, 85 \mathrm{ACV}$, y 50 casos de ICC. Los eventos cardiovasculares se presentaron con una incidencia creciente a medida que se avanza de categoría tensional. La incidencia acumulativa a 10 años de enfermedad cardiovascular en pacientes de 35 a 64 años con PA normal alta fue del $4 \%$ para mujeres (IC95\% 2 a 5), y del $8 \%$ para hombres (IC95\% 6 a 10). En ancianos de 65 a 90 años, la incidencia fue del $18 \%$ (IC95\% 12 a 23) para mujeres, y $25 \%$ (IC95\% 17 a 34) en hombres. Comparando con PA óptima, la PA normal alta mostró un riesgo relativo de 2,5 (IC95\% 1,6 a 4,1) en mujeres, y de 1,6 en hombres (IC95\% 1,1 a 2,2).

\section{Conclusiones}

La presión arterial normal alta está asociada a un mayor riesgo de enfermedad cardiovascular.

Fuente de financiamiento: National Heart, Lung and Blood Institute, EE.UU.

\section{Comentario}

Existe sólida evidencia que sustenta la recomendación de tratar individuos hipertensos con PA sistólica superior a $140 \mathrm{~mm} \mathrm{Hg}$, y/o PA diastólica mayor de $90 \mathrm{~mm} \mathrm{Hg}$. Sin embargo, sabemos que la incidencia de eventos cardiovasculares es creciente con los niveles de presión arterial, incluso por debajo de esos umbrales ${ }^{`}$. El presente trabajo muestra esa mayor incidencia de enfermedad cardiovascular en pacientes con registros basales de presión superiores a los óptimos, aún sin ser hipertensos. Sin embargo cabe realizar algunas observaciones. Recordemos que entre las limitaciones de los estudios de cohortes se encuentra su relativa ineficiencia para la evaluación de los eventos raros, y por definición a menores niveles de PA, menor frecuencia de eventos cardiovasculares. Cuando el estudio es retrospectivo, requiere registro de datos adecuado y completo. En este sentido la categorización de los pacientes se basó solo en un registro basal de presión arterial cuya variabilidad, y más en las cifras borderline que estamos analizando, puede llevar a una clasificación equívoca de un porcentaje de los individuos. Además, los pacientes con PA normal alta eran de mayor edad, mayor peso y tenían niveles de colesterolemia más elevados que los de PA óptima. Si bien el análisis se realizó con el ajuste de estas variables, podrian existir otras fuentes potenciales de sesgo no controladas en el análisis. La principal limitación de los estudios observacionales es el potencial sesgo resultante de la pérdida de casos en el seguimiento. Si las pérdidas son numerosas, o incluso escasas pero relacionadas tanto al factor de riesgo como al evento esperado, los resultados del estudio pueden ser cuestionados ${ }^{2}$. Por ejemplo en este estu- dio, antes de la selección definitiva de los casos, 408 paciente (3\%) fueron excluidos porque la información sobre las variables utilizadas en el análisis multivariable* era incompleta. Más allá de las consideraciones metodológicas, nos resta el impacto clínico de los hallazgos. Si bien los resultados son consistentes con la literatura previa en cuanto al riesgo creciente de enfermedad cardiovascular a medida que aumenta la presión arterial, resta comprobar el beneficio con el tratamiento en pacientes con registros de presión arterial normal alta. El estudio HOT ${ }^{3}$, que investigó los efectos del tratamiento óptimo de hipertensos con PAD entre 100 y $115 \mathrm{~mm} \mathrm{Hg}$ llevándolos con tratamiento farmacológico a PAD < $90,<85$ o < 80 , concluyó que por debajo de $85 \mathrm{~mm} \mathrm{Hg}$ las diferencias en eventos vasculares no serían significativas. De hecho no descartó una curva tipo $J$ ya que pacientes con PAD media de $86 \mathrm{~mm} \mathrm{Hg}$ tuvieron menor mortalidad cardiovascular que los que alcanzaron una PAD de 82. Los beneficios linealmente crecientes con el descenso de la PAD se registraron solo en poblaciones seleccionadas como los pacientes diabéticos.

Conclusiones del comentador: Queda claro que con e aumento de los niveles de presión arterial aumenta el riesgo de eventos cardiovasculares, aún en niveles considerados por debajo del umbral actual de hipertensión. Sin embargo se requiere de ulteriores estudios para recomendar la intervención en pacientes con cifras tensionales inferiores a 140/90, especialmente si se trata de pacientes con HTA no complicada y sin comorbilidad.

Dr. Alejandro Regueiro [ Grupo de Evaluación de Tecnologias. Superinntendencia de Servicios de Salud ]

Referencias

1 Blood pressure as a cardiovascular risk factor: prevention and treatment. Kannel WV. Jama 1996: 275: 1571-1576

2. Epidemiology in Medicine. Hennekens C. Buring J. Capitulo 7: 168-174. Little, Brown and Co. Boston/ Toronto. 1 ra Edición, 1987.

3. Effects of intensive blood-pressure lowering and low-dose aspirin in patients with hypertension: principal results of the Hypertension Optimal Treatment (HOT) randomised trial. HOT Study Group. Hanson L, Zanchetti A, et al. Lancet. 1998 Jun 13; 351:1755-62.

4.Long-term absolute benefit of lowering blood pressure in hypertensive patients according to the JNC VI risk stratification. Ogden LG, He J, et al. Hypertension 2000; 35: 539-543 